\title{
Level of radiation dose in university hospital non- insured private health screening programs in Korea
}

\author{
Yun-Keun Lee \\ Wonjin Institute for Occupational and Environmental Health, Seoul, Korea
}

\begin{abstract}
Objectives The aim of this study is to evaluate radiation exposure resulting from the comprehensive health examinations of selected university hospital programs and to present basic data for research and management strategies on the health effects of medical radiation exposure.

Methods Radiation-based diagnostic studies of the comprehensive health examination programs of ten university hospitals in Seoul, Korea, as introduced in their websites, were analyzed. The medical radiation studies of the programs were reviewed by radiologists. Only the effective doses of the basic studies were included in the analysis. The optional studies of the programs were excluded.

Results Among the 190 comprehensive health examination programs, 132 programs $(69.5 \%)$ included computed tomography studies, with an average of 1.4 scans. The average effective dose of radiation by program was $3.62 \mathrm{mSv}$ for an intensive program for specific diseases; $11.12 \mathrm{mSv}$ for an intensive program for cancer; $18.14 \mathrm{mSv}$ for a premium program; and $24.08 \mathrm{mSv}$ for an overnight program. A higher cost of a programs was linked to a higher effective dose $(r=0.812)$. The effective doses of the examination programs for the same purposes differed by as much as 2.1 times by hospital. Inclusion of positron emission tomography-computed tomography was the most critical factor in determining the level of effective dose.

Conclusions It was found that radiation exposure dose from comprehensive health exam programs targeted for an asymptomatic, healthy public reached between 3.6 and 24 times the annual dose limit for the general public. Relevant management policies at the national level should be provided to minimize medical radiation exposure.
\end{abstract}

Keywords Non-insured commercial health screening program, Computed tomography, Medical radiation

\section{Introduction}

Medical radiation refers to radiation produced during the diagnosis or treatment of disease. The radiation dose that is generated per study over the course of diagnosis, although differing by modality, can exceed the annual dose limit for the general public of $1.0 \mathrm{mSv}$ by up to a dozen times. For example, abdominal/pelvic computed tomography (CT), a commonly used exam in health diagnosis, may expose one to $10 \mathrm{mSv}$ of radiation per scan [1]: A single CT scan exceeds the annual dose limit for the general public by more than ten times, which is 3.3 times the to-
Correspondence: Yun-Keun, Lee

53 Sagajeong-ro 49-gil, Jungnang-gu, Seoul 02221 , Korea

Tel: $+82-2-490-2088$

Fax: $+82-2-490-2099$

E-mail:lyk4140@hanmail.net

Received: December 24, 2015

Accepted: March 22, 2016

Published: March 24, 2016

This article is available from: http://e-eht.org/ 
radiation exposure dose. According to recently published statistics by the Organization for Economic Cooperation and Development (OECD) [4], the number of CT modalities provided in Korea also showed a two-fold increase in 2013 (7298133 units) compared to six years ago. The number of CT scans per 1000 population is 132.3 in Korea, the seventh highest level among OECD members. The data clearly shows that Korea provides an environment where one is highly likely to be exposed to radiation from CT scans.

As CT exams are known to be the main source of medical radiation exposure, many studies have been conducted on the risk of cancer caused by radiation exposure [1,5,6]. Brenner et al. [1] argued that $1.5 \%$ to $2.0 \%$ of cancer cases occurring in the US were due to radiation exposure from CT scans. Another study that followed up on the cancer occurrence rates of 178 thousand children and young adults who had CT scans before the age of 22 revealed that the subjects' occurrence risk for leukemia (cumulative radiation dose of $30 \mathrm{mGy}$ or higher) and brain tumor (cumulative dose $60.42 \mathrm{mGy}$ ) were 3.18 times and 2.82 times higher, respectively, than unexposed people [5].

Health examinations are essential to detect disease in early stages to preserve health. However, some health examination programs are known to carelessly perform CT exams with high radiation exposure in order to rule out other conditions. Among such programs, comprehensive health check-up programs for intensive examinations in particular, mainly involve CT scans on multiple body parts simultaneously, which leads to relatively higher risk of cumulative radiation exposure.

According to a Korea Health Industry Development Institute report in 2013, about 952070 people receive comprehensive health examinations annually in medical institutions at university hospital level or above in Korea. Among them, 31.2\% (296744) are known to receive tests at university hospitals with 800 beds or more, and $56.8 \%$ (540579) do so at general hospitals with 400 beds to 800 beds [7]. As bigger hospitals tend to charge more for comprehensive health exams, their programs are very likely to include $\mathrm{CT}$ and therefore carry more risk of cumulative radiation exposure.

However, studies of medical radiation to date have focused on radiation exposure from individual tests, including CT scans. Lit- tle has been studied on the radiation exposure of comprehensive health examination programs consisting of various radiation tests.

This study evaluated radiation exposure doses from different comprehensive health examination programs offered by ten university hospitals in Korea. The aim of this study is to provide basic data for research on health and management strategies to limit medical radiation exposure.

\section{Materials and Methods}

\section{Subjects}

Radiation studies of comprehensive health examination programs as introduced in the websites of ten university hospitals based in Seoul were analyzed. The results revealed that there were 35 of the most common, basic programs; 70 disease-specific programs, such as for cardiovascular diseases, hypertension, or diabetes (excluding cancer diagnosing programs); 35 programs for intensive programs for cancer; 26 premium programs for diagnosing specific diseases and cancers; and 24 overnight programs consisting of almost all the tests needed for all types of diseases. There was a total of 190 programs. .

With regards to the radiation studies by examination program, basic examinations included a chest X-ray, and a bone density test and a mammography were added for females (Table 1). Disease-specific tests included coronary CT angiography or abdominal CT, depending on the cardiovascular diseases that were the primary purpose of the test. Intensive programs for cancer may include abdominal/pelvic CT, low dose chest CT, or a coronary calcium scan on top of the basic studies. Some hospitals also add positron emission tomography-computed tomography (PET-CT). Premium exams are often composed of intensive examinations for cancer and studies for specific diseases. Coronary artery CT or PET-CT scans may be included depending on the hospital. Overnight exam programs were the most costly, usually including studies of premium programs and PET-CT.

\section{Calculating Effective Dose}

Effective dose by radiation modality is based on the material on effective doses by modality in Korea, which is published by the Korea Institute of Nuclear Safety (Table 2) [8]. The effec-

Table 1. Diagnostic radiology procedures by private health screening program type

\begin{tabular}{lll}
\hline Health screening program & Examination items & \\
\hline Basic & Chest X-ray, bone densitometry (female), mammography (female) & Optionally determined by the \\
Specific disease (heart, stroke, brain, etc.), & Basic screening + dental panorama, low dose lung CT, & hospital \\
$\begin{array}{l}\text { cancer, premium (specific disease \& cancer), } \\
\text { hotel examination }\end{array}$ & abdomen/pelvis CT, calcium scoring CT, coronary angiography, & \\
\hline
\end{tabular}

CT, computed tomography; PET-CT, positron emission tomography-computed tomography. 
tive doses of other studies such as a low dose chest CT [9], coronary calcium scan [10], coronary angiography [10], or PETCT [11] were based on individual research results.

Whether the studies included in each examination produced radiation was determined through a review of laboratory medicine physicians. Only the effective doses of basic tests were analyzed, and optional studies in the programs were excluded. Also, for the chest CT, the effective dose of a low dose CT was used. In cases where a program included two or more CT tests, the effective doses were calculated based on the assumption that the scans were taken separately on different body parts. Therefore, the effective doses may be higher or lower depending on the types of CT test actually chosen in the hospital, optional studies chosen by the examinee, and how the scan was taken (e.g., scanning adjacent body parts together).

\section{Results}

\section{Number of Computed Tomography Scans}

The number of CT scans taken, the most critical variable in radiation exposure from studies in comprehensive exam programs, was analyzed per program (Table 3 ). The results revealed

Table 2. Adult effective doses for various diagnostic radiology and CT procedures

\begin{tabular}{llcc}
\hline & Examination & Average effective dose (mSv) & Reference \\
\hline Radiology & Bone densitometry & 0.004 & 8 \\
& Teeth panoramic & 0.01 & \\
Chest & 0.02 & \\
Cervical spine & 0.07 & \\
Thoracic spine & 0.7 & \\
Mammography & 0.27 & 8 \\
LT & 1.0 & 8 \\
& Lumbar spine & 2.0 & 9 \\
& Head & 10.0 & 10 \\
& Abdomen, pelvis & 1.14 & 10 \\
& Low dose lung & 3.0 & 11 \\
Calcium scoring & 16.0 & \\
& Coronary angiography & 14.5 & \\
PET & &
\end{tabular}

$\mathrm{CT}$, computed tomography; PET, positron emission tomography.

Table 3. Number of computed tomography (CT) scans by private health screening program type

\begin{tabular}{lcccc}
\hline \multirow{2}{*}{ Health screening program } & $\mathrm{n}$ & \multicolumn{3}{c}{ No. of CT scan check-up } \\
\cline { 3 - 5 } & & Mean & Minimum & Maximum \\
\hline Basic & 35 & 0.2 & 0 & 1 \\
$\begin{array}{l}\text { Specific disease } \\
\quad \text { (heart, stroke, brain, etc.) }\end{array}$ & 70 & 0.7 & 0 & 2 \\
$\begin{array}{l}\text { Cancer } \\
\text { Premium }\end{array}$ & 35 & 1.8 & 0 & 3 \\
$\quad$ (specific disease \& cancer) & 26 & 2.9 & 2 & 3 \\
Hotel examination & 24 & 3.5 & 3 & 4 \\
Total & 190 & 1.4 & 0 & 4 \\
\hline
\end{tabular}

that five programs (14.3\%) out of the 35 included CT tests, all of which were low dose chest CT with relatively low effective doses. In contrast, exam programs with higher costs such as intensive programs for cancer, premium programs, or overnight programs, tended to offer more CT scans. An intensive program for cancer on average had 1.8 CT scans per program. There were 24 intensive programs for cancer $(68 \%)$ that included abdominal CTs - with relatively greater radiation exposure (10 $\mathrm{mSv} / \mathrm{study}$ ) - out of the total of 35 programs. A premium program had, on average, 2.9 times more CT scans, with a very high ratio of abdominal CT scans (92.3\%). An overnight program, the most expensive type, included 3.5 CT scans on average. As high as $83.3 \%$ of the programs provided a PET-CT, which produces high radiation exposure.

\section{Effective Dose}

The results of the calculation of effective dose per examination program are demonstrated in Table 4. Basic programs showed the lowest effective dose ( $0.33 \mathrm{mSv}$ on average), mainly from a chest X-ray and mammography (for females). The average effective dose of intensive programs for specific diseases was 3.62 $\mathrm{mSv}$, with a peak of $13.3 \mathrm{mSv}$. Effective doses of this type of program ranged greatly, as a coronary CT or PET-CT test was selectively included depending on disease type. Intensive programs for cancer were usually comprised of an abdominal/pelvic $\mathrm{CT}$, low dose chest $\mathrm{CT}$, and a coronary calcium scan. PET$\mathrm{CT}$ was added in some hospitals. The average effective dose of the programs was $11.12 \mathrm{mSv}$, a relatively high level.

Premium examinations commonly consisted of intensive examinations for cancer and specific diseases. The average effective dose was $18.14 \mathrm{mSv}$ and a peak at $26.19 \mathrm{mSv}$, depending on the added studies. Overnight programs, the most costly type, frequently included a PET-CT in addition to premium program studies. Its effective dose averaged $24.08 \mathrm{mSv}$, the highest of all types of programs. The maximum dose was as high as $30.97 \mathrm{mSv}$.

Also analyzed was whether there was a difference in effective

Table 4. Comparison of effective doses by private health screening program type

\begin{tabular}{lrccr}
\hline Health screening program & Mean & SD & Min & Max \\
\hline Basic & 0.33 & 0.52 & 0.02 & 1.71 \\
$\begin{array}{l}\text { Specific disease } \\
\quad \text { (heart, stroke, brain, etc.) }\end{array}$ & 3.62 & 4.03 & 0.02 & 13.30 \\
Cancer & 11.12 & 3.79 & 1.44 & 16.19 \\
Premium & 18.14 & 4.89 & 4.43 & 26.19 \\
$\quad$ (specific disease \& cancer) & & & & \\
Hotel examination & 24.08 & 6.35 & 14.45 & 30.97 \\
Total & 8.46 & 8.61 & 0.02 & 30.97 \\
\hline
\end{tabular}

Unit: mSv.

SD, standard deviation; Min, minimum, Max, maximum. 


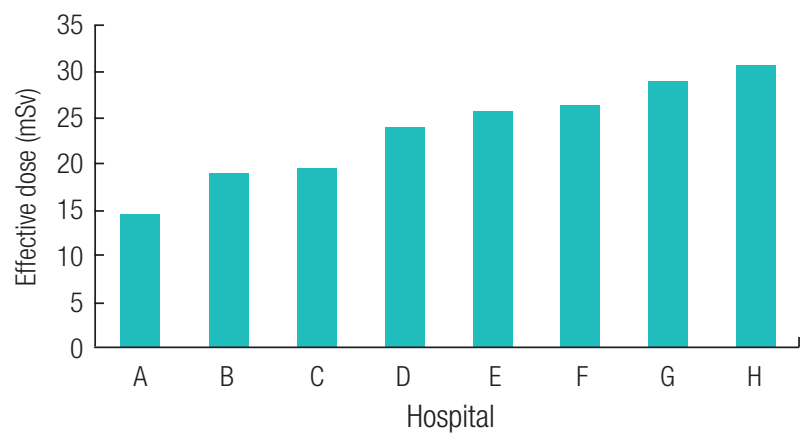

Figure 1. Comparison of the effective dose of private hotel examinations by hospital.

dose among the different hospitals in comparable exam programs for similar purposes (Figure 1). Overnight programs composed of the prime studies essential for disease diagnosis were analyzed. The effective doses of a total of eight hospitalsexcluding two university hospitals without overnight programs-were compared.

The results of the analysis showed that the lowest effective dose a hospital produced was $14.6 \mathrm{mSv}$, compared to the highest dose of $30.8 \mathrm{mSv}$, with a gap of more than 2.1 times. Hospitals with higher effective doses mostly included PET-CT, signifying that the inclusion of PET-CT was the biggest determinant of effective dose.

Also analyzed was how examination costs can make a difference in terms of effective dose (Figure 2). The result showed that higher costs led to an increase in effective dose, with a very strong association (correlation coefficient of 0.812 )

\section{Discussion}

To date, medical radiation has been widely used without much scrutiny. This is because the benefits gained from these tests or treatments using radiation outweigh concerns about radiation exposure. However, in some cases, people fall victim to random exposure to radiation through unnecessary, duplicate tests in the diagnosing process, or through comprehensive health examinations that repeat each year.

Government statistics show that as of 2011, the number of CT scans taken annually in Korea is about six million [3]. Among the examinees, as many as 507423 retook the exam by transferring to another hospital. Furthermore, among them, 99190 (19.5\%) received tests on the same body part again within 30 days after the first scan. Accordingly, it is possible that a significant portion of examinees may be exposed to unnecessary, duplicate radiation. As CT scans show high contribution rates to medical radiation exposure, many reports in the literature have pointed to these tests as the main source of medical radiation

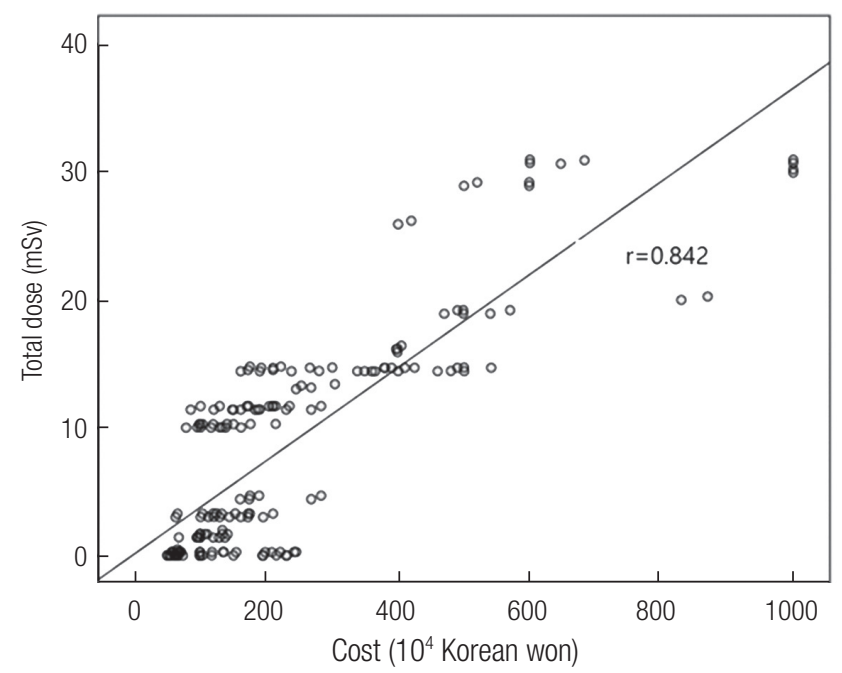

Figure 2. Relationship between private health screening cost and effective dose.

exposure $[1,3,5]$.

Reportedly, the ratio of CT scans in total medical radiation exposure in the population of Korea is $56.4 \%$, slightly higher than the overall average in European countries (52\%) [3]. According to the results of an analysis conducted in Europe, the average per capita medical radiation exposure dose in EU countries is 1.10 $\mathrm{mSv}$. The ratio represented by CT scans was the largest (52\%), followed by general radiography (22\%), and fluoroscopic exams (13\%) [12].

The results of this study demonstrated that $69.5 \%$ of examination programs included CT scans on one or more body parts. Notably, tests with higher costs included more CT studies and caused greater radiation exposure. This number of $\mathrm{CT}$ tests and the exposure doses produced were from university hospitals whereas the number of CT tests may be fewer in smaller-sized hospitals. Therefore, the results have limitations to be generalized to all comprehensive health examination programs.

The results of a study conducted in Korea [13] showed that the contribution to radiation exposure made by CT tests in comprehensive health examination programs was $72.1 \%$, and smaller-sized hospitals were likely to produce less radiation. These results are consistent with the findings of previous research $[1,3,5,13]$ that CT tests account for the largest share in generating medical radiation. Therefore, considering the composition of comprehensive examination programs, which mostly include CT scans, the issue of random radiation exposure from comprehensive programs should be seen as a high priority in order to reduce radiation exposure to the general public.

To date, many studies have been done on the risk of cancer occurrence due to medical radiation, in particular, from CT scans. According to the results of a study conducted in Korea [14], a 
dynamic abdominal CT scan produces $24.52 \mathrm{mSv}$, and the lifetime attributable risk of cancer per 100 thousand population is 220.8 people for males and 335.6 for males. A level of $24.5 \mathrm{mSv}$ is equal to the average effective dose of overnight programs as evaluated in this study.

Sodickson et al. [15] in their study of cancer occurrence of patients who have undergone one or more CT exams, reported that $15 \%$ of patients were exposed to a cumulative dose of radiation $100 \mathrm{mSv}$ or higher, and $4 \%$ were exposed to between 250 and $1375 \mathrm{mSv}$. In addition, the cancer occurrence rate of the patients followed by radiation exposure was $0.7 \%$, and their cancer mortality rate was about $1 \%$. A cumulative dose of $100 \mathrm{mSv}$ is equivalent to the dose built up through undergoing overnight programs provided by university hospitals four times.

Cancer occurrence due to radiation exposure is known to vary by sex and age. According to Bingsheng et al. [16], a PET-CT ( 13.45 to $13.65 \mathrm{mSv}$ ) is evaluated to cause cancer in 328 males and 362 females per 100 thousand population in their 20s, putting females at higher risk than their male counterparts. For males, the cancer occurrence rate in the $20 \mathrm{~s}$ is about 1.5 times higher than that in the 40s, around 1.7 times that in the 60s, and about 3.5 times that in the $80 \mathrm{~s}$.

In the US, about 29 thousand people are annually diagnosed with cancer resulting from CT scans. A third of them are between the ages of $35 \%, 54 \%$, and $66 \%$ of them are estimated to be female [17]. As such, cancer occurrence rates caused by medical radiation should not be underestimated. Many countries have determined that, despite the great benefits of CT scans, the potential risk of the test must become better understood [18]. In Korea, it has been reported that people without specific symptoms increasingly receive CT tests only for the purpose of disease screening [3]. Such reckless use of CT tests cannot be justified and thus the basic principle of radiation protection, "As low as reasonably achievable," must be followed [19].

The International Commission on Radiological Protection suggests that patients who are undergoing disease screening processes should be sufficiently informed of the risk of radiation, not just its potential benefits [20]. To educate the public, the International Atomic Energy Agency has staged an "AAA" initiative to minimize medical radiation exposure. This effort promotes the triple "A" framework: accurate awareness of radiation risk by doctors, relevant staff, and patients (Awareness); appropriate performance of tests under proper circumstances (Appropriateness); and constant management and audits to check if the above factors have been duly placed (Audit) [21].

Medical radiation can be sufficiently reduced through accurate information and management standards. Since 1992, radiation studies in the UK have been conducted by considering yearly exposure dose, making calculations, and recording patients' mandatory exposed dose (UK National Patient Dose Database) [22]. Moreover, the state of California mandated in law that all institutions using $\mathrm{CT}$ scans document patient radiation exposure dose and provide the data annually in case a relevant organization requests them [23].

Australia minimizes medical radiation exposure through the National Diagnostic Imaging Accreditation Scheme, an organizational system for patient radiation exposure management [24]. In order to ban unjustified medical radiation exposure, the EU requires its members to establish relevant law and introduce a managing and supervising system [25].

In contrast, in most parts of the US, except for some states, there is no management standard and therefore the medical radiation exposure dose is reported as increasing. Due to this policy gap, the country saw a large hike in the annual medical radiation exposure dose from $0.53 \mathrm{mSv}$ in the $1980 \mathrm{~s}$ to $3.0 \mathrm{mSv}$ in 2006. In comparison, the figure in the $\mathrm{UK}$ is $0.4 \mathrm{mSv}$, significantly lower than that of the US [26], and less than $30 \%$ of the average per capita exposure dose in Korea $(1.4 \mathrm{mSv})$.

As seen in the case of the UK, medical radiation is common artificial radiation that can be reduced by government policy. Therefore, if policies intended to lower radiation exposure are implemented in Korea, the country would be able to minimize the dose.

The results of this study show that medical radiation exposure doses from comprehensive health examination programs are at a serious level, enough to significantly influence an increase in cancer occurrence. Therefore, the establishment of law and adoption of managing systems to regulate unnecessary radiation exposure must be a top priority. In terms of management, guidelines for disease diagnosis such as through comprehensive examinations are needed. Also, periodical evaluation of exposure dose from radiation devices and maintenance of modalities as well as development of protocols for minimizing radiation dose from study devices are also necessary.

\section{Acknowledgements}

This study was funded by the Korea Environmental Industry and Technology Institute, Ministry of Environment (no. 412-111008).

\section{Conflict of Interest}

The author has no conflicts of interest associated with material presented in this paper. 


\section{ORCID}

Yun-Keun Lee http://orcid.org/0000-0001-6090-7665

\section{References}

1. Brenner DJ, Hall EJ. Computed tomography--an increasing source of radiation exposure. N Engl J Med 2007;357(22):2277-2284.

2. Korea Institute of Nuclear Safety. Assessment of radiation risk for the Korean population. Daejeon: Korea Institute of Nuclear Safety; 2007, p. 186 (Korean).

3. Ministry of Food and Drug Safety. The national status of medical radiation effective dose per year: 2007-2011; 2014 [cited $2015 \mathrm{Nov}$ 23]. Available from: http://www.mfds.go.kr/index.do?seq= $22654 \&$ mid $=675$ (Korean).

4. Organization for Economic Cooperation and Development. Health status; 2015 [cited 2015 Nov 23]. Available from: http:// stats.oecd.org/index.aspx?DataSetCode=HEALTH_STAT.

5. Pearce MS, Salotti JA, Little MP, McHugh K, Lee C, Kim KP, et al. Radiation exposure from CT scans in childhood and subsequent risk of leukemia and brain tumors: a retrospective cohort study. Lancet 2012;380(9840):499-505.

6. Huang B, Law MW, Khong PL. Whole-body PET/CT scanning: estimation of radiation dose and cancer risk. Radiology 2009; 251(1):166-174.

7. Korea Health Industry Development Institute. Healthcare industry survey for aging. Cheongju: Korea Health Industry Development Institute; 2013, p. 91(Korean).

8. Korea Institute of Nuclear Safety. Design and construction of radiation dose database for Korean population. Daejeon: Korea Institute of Nuclear Safety; 2005, p. 178-179 (Korean).

9. Larke FJ, Kruger RL, Cagnon CH, Flynn MJ, McNitt-Gray MM, $\mathrm{Wu} \mathrm{X}$, et al. Estimated radiation dose associated with low-dose chest CT of average-size participants in the National Lung Screening Trial. AJR Am J Roentgenol 2011;197(5):1165-1169.

10. Mettler FA Jr, Huda W, Yoshizumi TT, Mahesh M. Effective doses in radiology and diagnostic nuclear medicine: a catalog. Radiology 2008;248(1):254-263.

11. Willowson KP, Bailey EA, Bailey DL. A retrospective evaluation of radiation dose associated with low dose FDG protocols in wholebody PET/CT. Australas Phys Eng Sci Med 2012;35(1):49-53.

12. European Commission. Medical radiation exposure of the European population; 2014 [cited $2016 \mathrm{Feb} 23$ ]. Available from: https:// ec.europa.eu/energy/sites/ener/files/documents/RP180.pdf.

13. Kim HJ, Kim JW, Lee SH, Kim JY, Kim MY. Radiation exposure through private health examinations in Korea. Korean J Health Promot 2015;15(3):136-140 (Korean).

14. Kang YH, Park JS. Radiation dose and lifetime attributable risk of cancer estimates in 64-slice multidetector computed tomography. J
Korea Contents Assoc 2011;11(4):244-252 (Korean).

15. Sodickson A, Baeyens PF, Andriole KP, Prevedello LM, Nawfel $\mathrm{RD}$, Hanson R, et al. Recurrent CT, cumulative radiation exposure, and associated radiation-induced cancer risks from CT of adults. Radiology 2009;251(1):175-184.

16. Huang B, Ming Law MW, Khong.PL. Whole-body PET/CT scanning: estimation of radiation dose and cancer risk. Radiology 2009; 251(1);166-174

17. Berrington de González A, Mahesh M, Kim KP, Bhargavan M, Lewis R, Mettler F, et al. Projected cancer risks from computed tomographic scans performed in the United States in 2007. Arch Intern Med 2009;169(22):2071-2077.

18. Albert JM. Radiation risk from CT: implications for cancer screening. AJR Am J Roentgenol 2013;201(1):W81-W87.

19. Chawla SC, Federman N, Zhang D, Nagata K, Nuthakki S, McNittGray $M$, et al. Estimated cumulative radiation dose from PET/CT in children with malignancies: a 5-year retrospective review. Pediatr Radiol 2010;40(5):681-686.

20. International Commission on Radiological Protection (ICRP). ICRP Publication 105. Radiation protection in medicine. Ann ICRP 2007;37(6):1-63.

21. International Atomic Energy Agency (IAEA). Triple-A investment in patients' health: IAEA promotes awareness, appropriateness, audit of ionizing radiation in medicine; 2010 [cited 2016 Feb 23]. Available from: https://www.iaea.org/newscenter/news/triple-investment-patients-health.

22. Hart D, Hillier MC, Shrimpton PC. Doses to patients from radiographic and fluoroscopic X-ray imaging procedures in the UK-2010 review (HPA-CRCE-034); 2012 [cited 2016 Mar 12]. Available from: http:// webarchive.nationalarchives.gov.uk/20140714084352/ http://www. hpa.org.uk/webc/HPAwebFile/HPAweb_C/ 1317134577210.

23. California Department of Public Health. Information notice regarding Senate Bill (SB) 1237, California Health and Safety (H\&S) Code Section 115113; 2011 [cited 2016 Mar 13]. Available from: https://www.cdph.ca.gov/certlic/radquip/Documents/RHBSB1237-FAQ.PDF.

24. Australian Radiation Protection and Nuclear Safety Agency. Radiation protection in diagnostic and interventional radiology; 2008 [cited 2016 Feb 23]. Available from: http://www.arpansa.gov.au/ pubs/rps/rps14_1.pdf.

25. European Commission. Council directive 2013/59/EURATOM; 2013 [cited 2016 Feb 23]. Available from: http://eur-lex.europa. eu/LexUriServ/LexUriServ.do?uri=OJ:L:2014:013:0001:0073:E N:PDF.

26. United Nations. Sources and effects of ionizing radiation. Volume 1: UNSCEAR 2008 report to the General Assembly with scientific annexes; 2010 [cited 2016 Feb 23]. Available from: http://www. unscear.org/docs/reports/2008/09-86753_Report_2008_Annex_B.pdf. 